\section{Drohende Grippeepidemie in Berlin?}

Das Hauptgesundheitsamt hatte am 5. I. zu einer Besprechung in kleinerem Kreise einige Dirigierende Aerzte der Städtischen Krankenhäuser und charitativer Anstalten, Vertreter des Groß-Berliner Aerztebundes, einen Vertreter der Pathologischen Anatomie und einen Hals-Nasen-Ohrenspezialisten gebeten. $\mathrm{Z}$ we if ellos beindet sich Berlin im Beginn einer sogenannten Grippewelle. Da die bakteriologische Diagnose für Erkennung und Bekämpiung der "Grippe“ dem Arzt am Krankenbett keine Stütze geben kann, muß, wie auch früher, das klin ische Bild ausschlaggebend sein. Alle Anwesenden waren sich darüber einig, daß neben der Häufung katarrhalischer Saisonerkrankungen grippöse Krankheitsbilder in zunehmender Zahl auftreten. So konnte das Hauptgesundheitsamt Zahlen aus den Krankenhäusern und aus der Allgemeinen Ortskrankenkasse Berlin mitteilen, die bei aller Kritik ein deutliches Anschwellen grippöser Erkrankungen in der zweiten Dezemberhälfte zeigen. Auch muß schon hie und da ein Auftreten leicht toxischer Krankheitsbilder, was bisher noch nicht der Fall war, vermerkt werden. Die Verbindung mit Pneumonien bat sich in den letzten Tagen gehäuft, es wurde sogar eine auffällige Zunahme pleuritischer Komplikationen besprochen; jedoch erinnerten diese Bilder bisher durchaus nicht an die schweren toxischen Zustände der sogenannten „spanischen Grippe“ aus der Kriegs- und Nachkriegszeit. Wohl sind vereinzelt autoptische Befunde schwerer Veränderungen an den Schleimhäuten des Respirationstraktus festgestellt worden, die dem typischen Bild grippöser Infektionen entsprechen. Hämorrhagische Erkrankungen des Ohres liegen bisher ebenfalls nur ganz vereinzelt vor; neurotoxische Beobachtungen fehlen.

Da, wie aus früheren Epidemien bekannt, es nach allem nicht ausgeschlossen ist, daß sich in den nächsten Wochen das $\mathrm{K} \mathrm{r}$ a n $\mathbf{k}$ heitsbild, unter Umständen sogar sehr schnell, zu einem bedeutend schwereren entwickelt und daß auch der e pidemische Charakter sich verstärkt, wurde über Maßnahmen beraten, um dem Ansturm der Kranken auf die Krankenhäuser gerecht zu werden. Leider hat ein Teil der Tagespresse bereits eine wirklich noch unnötige Unruhe in die Bevölkerung Berlins getragen. Selbst zu therapeutischen Vorschlägen verstiegen sich gewisse Blätter. Eine sachgemäße und beruhigende Aufklärung durch den $\mathrm{R}$ u $\mathrm{n}$ f $\mathrm{u} \mathrm{nk}$ wurde aus der Versammlung dringend empiohlen. Besonders beachtenswert erschien der Hinweis, die Berliner Verkehrspolitik zu ändern, da die Sparmaßnahmen auch zu einer Ueberlastung der öffentlichen Verkehrsmittel geführt haben, die der Verbreitung gerade dieser infektiösen Erkrankung den besten Vorschub leistet. Leider sind alle Mahnungen durch Plakate in den Verkehrsmitteln erfolglos geblieben!

Wenn auch infolge der Notverordnungen und der wirtschaftlichen Schwierigkeiten sicher der Ansturm Kranker auf die Krankenhäuser gemildert sein wird, so muß doch genügend Bettenmaterial bereits jetzt zur Veriügung stehen, besonders wenn sich eine Periode der $\mathrm{K}$ omplikat i onen, wie ublich, anschließen wird. Berlin ist zur Zeit noch gewappnet, allerdings vorwiegend dadurch, daß in den letzten Wochen des vergangenen Jahres infolge einer auffallend geringen Belegung eine Anzahl von Abteilungen, Baracken, sogar ein kleineres Krankenhaus geschlossen werden konnten. Es besteht also infolgedessen eine Reserve, und es ist demnach für die Bereitstellung gesonderter „Grippestationen“, ebenso wie auch für „Rekonvaleszentenstationen“, die Möglichkeit.geschaffen.

Die Grippeist eine hochinfektiöse Erkrankung; Grippekranke auf den verschiedenen Stationen je nach Platzverhältnis unterzubringen, geht nicht an; sonst können bekanntlich, wie es auch bei früheren Epidemien stets zu beobachten war, die anderen Patienten durch eine Hausinfektion geschädigt werden. Es nuß auch an dieser Stelle, wie bereits früher (D. m. W. 1929 Nr. 4, 5, 7), hervorgehoben werden, daß Berlin trotz der namentlich von charitativen Institutionen in den letzten Jahren vorgenommenen Neubauten schon kleineren Epidemien gegenüber zweifellos nicht genügend mit einer Reservebettenzahl in seinen Krankenhäusern gerüstet ist. Selbst die ausgezeichnet funktionierende Mitarbeit der Aerzteschaft, die bestrebt ist, nur Schwerkranke dem Krankenhaus zu überweisen, selbst die rechtzeitige Verlegung von Rekonvaleszenten aus dem Krankenhaus in Leichtkrankenhäuser wird bei einem nur etwas stärkeren Anschwellen einer Epidemie nicht genügen, um die Kranken reibungslos unterzubringen. Man darf sich also nicht damit begnügen, daß v or e r s t die Krankenhausbetten ausreichen werden. Die Sparmaßnahmen von Berlin lkönnen sich unmöglich auf eine der kommunalen Hauptaufgaben: die hygienischen Maßnahmen, erstrecken: Ein Sportplatz ersetzt nicht ein Krankenhaus!

von den Velden. 